\title{
STRUCTURES DETERMINED BY PRIME IDEALS OF RINGS OF FUNCTIONS
}

\author{
BY \\ RICHARD G. MONTGOMERY( $\left.{ }^{1}\right)$
}

Introduction. Let $\mathscr{R}$ and $\mathscr{T}_{3 \frac{1}{2}}$ respectively denote the category of commutative rings with unity and the category of completely regular Hausdorff spaces; also, $\mathscr{C}$ denotes the full-subcategory of $\mathscr{T}_{3 \frac{1}{2}}$ whose objects are compact, and $\mathscr{C}_{T D}$ the full-subcategory of $\mathscr{C}$ whose objects are totally-disconnected. The collection of prime ideals of $A \in \mathscr{R}$ is the underlying set for an object $K_{A} \in \mathscr{C}_{T D}$ and $K$ is contravariantly functorial. If $C$ denotes the contravariant functor which assigns to each $X \in \mathscr{T}_{3 \frac{1}{2}}$ the ring $C(X)$ of real-valued continuous functions on $X$, then the resulting functor $K_{C}$ is the domain of a natural transformation $\tilde{\theta}: K_{C} \rightarrow \beta$, where $\beta$ denotes the Stone-Čech reflection of $\mathscr{T}_{3 \frac{1}{2}}$ into $\mathscr{C}$. The prime $z$-ideals of $C(X)$ also furnish such a space $\zeta X$, functor $\zeta$ and natural transformation $\theta: \zeta \rightarrow \beta$. In the appropriate category, $\zeta$ fills in a diagram which exhibits $\beta$ as a push-out.

Topological properties of $K_{C(X)}$ and $\zeta X$ are studied and $\zeta X$ is characterized as a certain compactification of $X_{P}$, the $\boldsymbol{P}$-topology on $X$, which helps establish the place of $\zeta X$ between $\beta X_{P}$ and $\beta X$.

The above results are applied in an investigation of the continuous and orderpreserved image of $\zeta Y$ in both of $\zeta X$ and $\beta X$ arising from $f: Y \rightarrow X$. As one consequence, the prime $z$-ideal structure and the minimal prime ideal structure associated with $X$ is illuminated by the corresponding structures associated with certain subspaces of $X$; as another, a convenient simplification and unification is provided for approaching several types of problems found in the literature on prime ideals of $C(X)$.

1. The functors $K_{C}$ and $\zeta$. If $A \in \mathscr{R}$, then $K_{A}$ denotes the collection of prime ideals of $A$ (which, in our usage, does not include $A$ itself). The following notations are used for a subset $S \subseteq A$ and $a \in A: \hat{h}(S)=\left\{P \in K_{A}: S \subseteq P\right\}, \tilde{h}^{c}(S)=K_{A} \mid \tilde{h}(S)$, $\tilde{h}(a)=\tilde{h}(\{a\})$ and $\tilde{h}^{c}(a)=\tilde{h}^{c}(\{a\})$. One can view $K_{A}$ as a subset of the product space $2^{A}$, where $2=\{0,1\}$ is the two-point discrete space; hereafter $K_{A}$ is endowed with its topology relative to $2^{A}$. Observing that $\tilde{h}(a)=\pi_{a}{ }^{-}[1] \cap K_{A}$ and $\tilde{h}^{c}(a)$ $=\pi_{a}{ }^{\leftarrow}[0] \cap K_{A}$, it follows that the collection $\{\tilde{h}(a): a \in A\} \cup\left\{\tilde{h}^{c}(a): a \in A\right\}$ is a

Received by the editors February 11, 1969.

(1) This paper is based on a portion of the author's doctoral dissertation written under the guidance of Professor John Kennison and supported by the National Science Foundation and Clark University. The author is grateful for valuable suggestions from Professors John Kennison and Norman Noble. 
subbase for $K_{A}$; moreover, since $\tilde{h}\left(a_{1}\right) \cap \ldots \cap \mathscr{h}\left(a_{n}\right)=\tilde{h}\left(\left\{a_{1}, \ldots, a_{n}\right\}\right)$ and $\tilde{h}^{c}\left(a_{1}\right) \cap \cdots \cap \tilde{h}^{c}\left(a_{n}\right)=\mathscr{h}^{c}\left(a_{1} \cdots \cdots a_{n}\right)$, we see that the collection $\left\{\mathscr{h}(S) \cap \mathscr{h}^{c}(a): S\right.$ is a finite subset of $A$ and $a \in A\}$ is a base for $K_{A}$.

If $S$ is a subset of $A$ which is not a prime ideal, then $S$ fails to satisfy one of a finite number of properties such as $a \in A, b \in S$ implies $a b \in S$; that is, there exist $a, b \in A$ with $S \in U=\pi_{b}{ }^{\leftarrow}[1] \cap \pi_{a b}{ }^{\leftarrow}[0]$. Whichever property does not hold for $S$, the corresponding $U$ provides a neighborhood of $S$ in $2^{A}$ which misses $K_{A}$. Hence, $K_{A}$ is closed in $2^{A}$ and $K_{A} \in \mathscr{C}_{T D}$.

The preceding argument clearly applies to commonly encountered collections of subsets in many concrete categories which are algebraic in nature; for more detail in this direction see $\left[\mathrm{M}_{3}\right]$.

$K_{A}$ deserves to be compared to $\operatorname{Spec} A$, the well-known [B] topology whose underlying set coincides with that of $K_{A}$. Since $\operatorname{Spec} A$ has the collection of $\mathscr{h}^{c}(a)$ $(a \in A)$ as a base, it is coarser than $K_{A}$; a fact which not only yields the known compactness of $\operatorname{Spec} A$ but also shows that $K_{A}=\operatorname{Spec} A$ if and only if every prime ideal of $A$ is maximal (as this is precisely when $\operatorname{Spec} A$ is Hausdorff). Spec is contravariantly functorial; specifically, $\operatorname{Spec} \alpha: \operatorname{Spec} B \rightarrow \operatorname{Spec} A$ is defined by $P \mapsto \alpha^{\leftarrow}[P]$ for $\alpha: A \rightarrow B$ in $\mathscr{R}$ and $P \in \operatorname{Spec} B$. Since Spec $\alpha$ is continuous by virtue of the equality $(\operatorname{Spec} \alpha)^{-}[\tilde{h}(a)]=\tilde{h}(\alpha(a))$, it easily follows that $K_{\alpha}: K_{B} \rightarrow K_{A}$ is also continuous, where $K_{\alpha}$ pointwise agrees with Spec $\alpha$. Using the functorial properties of Spec, we have the following

\subsection{LemmA. $K$ is a contravariant functor from $\mathscr{R}$ to $\mathscr{C}_{T D}$.}

Further relationships between $K_{A}$ and $\operatorname{Spec} A$ are found in $\left[\mathrm{M}_{3}\right]$; for present purposes, however, we immediately specialize to the case $A=C(X)$. Henceforth, an unmodified $X$ carries the assumption that $X \in \mathscr{T}_{3 \frac{1}{2}}$; also, $f: Y \rightarrow X$ indicates that $f$ is a continuous function from $Y \in \mathscr{T}_{3 \frac{1}{2}}$ to $X \in \mathscr{T}_{3 \frac{1}{2}}$. Notations not otherwise defined are found in [GJ].

Since $C: \mathscr{T}_{3 \frac{1}{2}} \rightarrow \mathscr{R}$ is contravariantly functorial, we have the functor $K_{C}: \mathscr{T}_{3 \frac{1}{2}} \rightarrow \mathscr{C}_{T D} ;$ specifically, for $f: Y \rightarrow X$ and $P \in K_{C(Y)}$,

$1.2 K_{C(f)}(P)=\{g \in C(X): g \circ f \in P\}$.

If $f: Y \rightarrow X$ and $g \in C(X)$, the obvious equality

$1.3 f^{\leftarrow}[Z(g)]=Z(g \circ f)$

is repeatedly used; in particular, it justifies (b) and (c) of the following proposition.

1.4 Proposition. $K_{C}$ is a functor from $\mathscr{T}_{3 \frac{1}{2}}$ to $\mathscr{C}_{T D}$. Moreover, if $f: Y \rightarrow X$, then:

(a) $K_{C(f)}$ preserves the ordering of set-inclusion;

(b) $\left[\mathrm{GJ}_{1}, 2.2\right] K_{C(f)}(P)$ is a (prime) $z$-ideal of $C(X)$ if $P$ is a prime $z$-ideal of $C(Y)$;

(c) $K_{C(f)}\left(M_{y}\right)=M_{f(y)}$ for $y \in Y$.

The space of our major interest is $\zeta X$, the collection of prime $z$-ideals of $C(X)$ topologized relative to $K_{C(X)}$. We let $\hat{f}$ denote $K_{C(f)}$ restricted to $\zeta Y$ if $f: Y \rightarrow X$; 
1.4(b) permits us to treat $\hat{f}$ as $\hat{f}: \zeta Y \rightarrow \zeta X$. If $S \subseteq C(X)$ and $g \in C(X)$, then $h(S)$ $=\tilde{h}(S) \cap \zeta X ; h^{c}(S), h(g)$ and $h^{c}(g)$ are similarly defined.

1.5 Proposition. The mappings $\zeta: X \mapsto \zeta X$ and $\zeta: f \mapsto \hat{f}$ define a functor $\zeta$ from $\mathscr{T}_{3 \frac{1}{2}}$ to $\mathscr{C}_{T D}$. Moreover, if $f: Y \rightarrow X$, then

(a) $\hat{f}$ is order-preserving;

(b) $\hat{f}\left(M_{y}\right)=M_{f(y)}$ for $y \in Y$.

2. Some topological properties of $\zeta X$ and $K_{C(X)}$. It is straightforward to verify that the category of $P$-spaces is coreflective in $\mathscr{T}_{3 \frac{1}{2}}$; specifically, let $X_{P}=\boldsymbol{P}(X)$ be the set $X$ topologized by taking the collection $Z(X)$ of zero-sets of $X$ as a base, and let $f_{P}=\boldsymbol{P}(f): Y_{P} \rightarrow X_{P}$ pointwise agree with $f: Y \rightarrow X$. Then $X_{P}$ is a $P$-space and the obvious map $p_{X}: X_{P} \rightarrow X$ is continuous; in fact, $p=\left\{p_{X}\right\}_{X \in \mathscr{T}_{3 \frac{1}{2}}}$ is the natural transformation from the coreflection functor $\boldsymbol{P}$ to $I_{3 \frac{1}{2}}$, the identity functor on $\mathscr{T}_{3 \frac{1}{2}}$. Moreover, $f_{P}$ is the unique continuous function $Y_{P} \rightarrow X_{P}$ with the property $p_{X} \circ f_{P}=f \circ p_{Y}$.

Note that since $Z(X)$ is closed under finite (even countable) intersections, the collection $\left\{H_{g, h}: g, h \in C(X)\right\}$ is a base for $\zeta X$, where $H_{g, h}=h(g) \cap h^{c}(h)$.

2.1 THEOREM. $\zeta X$ is a compactification of $X_{P}$. Specifically, the map $\nu_{X}: X_{P} \rightarrow \zeta X$ defined by $x \mapsto M_{x}\left(x \in X_{P}\right)$ is a homeomorphism onto the collection $\mathscr{M}_{F}(X)$ of fixed maximal ideals which is dense in $\zeta X$.

Proof. Since $Z(X)$ is a base for the space $X_{P}$ which is coarser than $X$ and $\nu_{X}^{\leftarrow}\left[H_{g, h}\right]=Z(g) \cap \operatorname{coz}(h)$, it follows that the one-to-one correspondence $\nu_{X}$ of $X_{P}$ onto $\mathscr{M}_{F}(X)$ is continuous; it is also open as $\nu_{X}[Z(g)]=h(g) \cap \mathscr{M}_{F}(X)$. Moreover, if $\varnothing \neq H_{g, h}$, then $Z(g) \ddagger Z(h)$ and $M_{x} \in H_{g, h}$ for each $x \in Z(g) \backslash Z(h)$.

Let $f: Y \rightarrow X$. Since $\nu_{Y}: Y_{P} \cong \mathscr{M}_{F}(Y)$ is dense in $\zeta Y, 1.5(\mathrm{~b})$ shows that $\hat{f}$ is the unique extension of $f_{P}$ to $\zeta Y$; consequently, we sometimes refer to $\hat{f}$ as the $\zeta$ extension of $f$ to $\zeta Y$. If $Y$ is a $P$-space, then $\zeta Y=K_{C(Y)}=\beta Y$; also, certain Stoneextensions are $\zeta$-extensions and hence have explicit (1.2) representations. One such extension is singled out in the following proposition. Henceforth the notation $\bar{f}$ will be reserved for the Stone-extension of $f: Y \rightarrow X$.

2.2 Proposition. If $f: Y \rightarrow X$, then $\hat{f} \circ \nu_{Y}=\nu_{X} \circ f_{P}$; that is, $\nu=\left\{\nu_{X}\right\}_{X \in \mathscr{F}_{3^{\frac{1}{2}}}}$ is a natural transformation from $\boldsymbol{P}$ to $\zeta$. Moreover, $\bar{\nu}_{X}=\hat{p}_{X}$; hence,

$$
\bar{\nu}_{X}(M)=\{g \in C(X): Z(g) \in Z[M]\}
$$

for $M \in \beta X_{P}$, and $\bar{\nu}_{X}\left(M_{1}\right)=\bar{\nu}_{X}\left(M_{2}\right)$ if and only if $Z(X) \cap Z\left[M_{1}\right]=Z(X) \cap Z\left[M_{2}\right]$.

Proof. The first statement follows from 1.5(b); the second holds as $\bar{\nu}_{X}$ and $\hat{p}_{X}$ each map $\zeta X_{P}=\beta X_{P}$ into $\zeta X$ and agree on dense $X_{P}$.

Note that $\bar{\nu}_{X}$ is onto $\zeta X$ (as $X_{P}$ is dense in $\zeta X$ ) and so is a quotient map; in fact, 2.2 shows that $\zeta X$ is the quotient of $\beta X_{P}$ under the equivalence relation: $M_{1} \sim M_{2} \Leftrightarrow Z\left[M_{1}\right] \cap Z(X)=Z\left[M_{2}\right] \cap Z(X)$. 
We call a point $x \in X$ a zero-set point of $X$ if $\{x\} \in Z(X)$. Note that $x$ is a zero-set point of $X$ if and only if $x$ is an isolated point of $X_{P}$.

A subspace of a $P$-space is itself a $P$-space [GJ, 4L] and a compact $P$-space is finite $[\mathrm{GJ}, 4 \mathrm{~K}]$. It follows that a $P$-space is locally compact if and only if it is discrete; also, it is $\sigma$-compact if and only if it is countable.

2.3 CoRollary.

(a) $X_{P}$ is open in $\zeta X$ if and only if either one of the following two (equivalent) conditions holds:

(1) $X_{P}$ is discrete;

(2) every point of $X$ is a zero-set point.

(b) $X_{P}$ is a cozero-set of $\zeta X$ if and only if either one of the following two (equivalent) conditions holds:

(1) $X_{P}$ is countable and discrete (that is, $X_{P} \cong N$ );

(2) $X$ is countable and every point of $X$ is a zero-set point.

(c) The isolated points of $\zeta X$ are precisely those of $X_{P}$; that is, $P$ is an isolated point of $\zeta X$ if and only if $P=M_{x} \in \mathscr{M}_{F}(X)$ and $x$ is a zero-set point of $X$.

Proof. A space $Y$ is open in any compactification $\bar{Y}$ if and only if it is locally compact [GJ, 3.15]; and is a cozero-set of $\bar{Y}$ if and only if it is locally compact and $\sigma$-compact (the latter condition from [GJ, 1.10]). Moreover, the isolated points of $Y$ and $\bar{Y}$ coincide [GJ, 3.15].

The isolated points of $K_{C(X)}$ are in one-to-one correspondence with the isolated points of $X$. Unfortunately, $K_{C(X)}$ has no convenient dense subset (as does $\zeta X$ ) and, before proceeding, we introduce the function $\tilde{\theta}_{X}: K_{C(X)} \rightarrow \beta X$ defined by $\tilde{\theta}_{X}(P)=p$, where $p$ is the unique [GJ, 7.15] point in $\beta X$ with $P \subseteq M^{p}$. It is shown in [HJ, 5.3] that $\tilde{\theta}_{X}$ restricted to the minimal prime ideals topologized relative to Spec $C(X)$ is continuous. The proof used there, however, readily adapts to show that $\tilde{\theta}_{X}$ is continuous when the domain space is $\operatorname{Spec} C(X)$; since $K_{C(X)}$ is finer than Spec $C(X)$, we have the continuity of $\tilde{\theta}_{X}: K_{C(X)} \rightarrow \beta X$.

2.4 TheOREM. A point $P$ of $K_{C(X)}$ is isolated if and only if $P=M_{x} \in \mathscr{M}_{F}(X)$ and $x$ is an isolated point of $X$.

Proof. Following [GJ, 14.2] we call a point in $K_{C(X)}$ an upper ideal if it has an immediate predecessor (in the ordering of set inclusion). Similarly, it is a lower ideal if it has an immediate successor.

Now let $Q$ be an isolated point of $K_{C(X)}$ and suppose the following two claims hold:

(a) If $Q$ is not maximal, then it is a lower ideal.

(b) If $Q$ is not minimal, then it is an upper ideal.

Then, if $Q \notin \zeta X$, it is neither maximal nor minimal [GJ, 14.7] and so is both an upper and a lower ideal ((a) and (b)), which is impossible [GJ, 14.18]. Hence, $Q \in \zeta X$ and so is isolated there; that is, $Q=M_{x} \in \mathscr{M}_{F}(X)$ and $x$ is a zero-set point 
of $X(2.3(\mathrm{c}))$. Since no prime $z$-ideal is an upper ideal [GJ, 14.10], $Q$ is also a minimal prime ideal (b); that is, $x$ is a $P$-point of $X$. At the same time, being a zero-set point, $x$ is a $G_{\delta}$-point of $X$. The conclusion now follows as every $P$-point $G_{\delta}$-point is isolated [GJ, $4 \mathrm{~L}$ ]. It remains to establish the claims.

Since $\{Q\}$ is open in $K_{C(X)}$, there exist $g_{1}, \ldots, g_{n}, h \in C(X)$ with $\{Q\}$ $=\left\{P \in K_{C(X)}: g_{1}, \ldots, g_{n} \in P\right.$ and $\left.h \notin P\right\}$. In what follows, the symbol $\subset$ denotes proper inclusion.

(a) If $Q \subset P \in K_{C(X)}$, then $g_{1}, \ldots, g_{n} \in P$ and so $h \in P$ (otherwise $P=Q$ ). Let $\bar{Q}=\bigcap_{Q \subset P} P$, then $\bar{Q} \in K_{C(X)}[\mathrm{GJ}, 14.2(\mathrm{a})$ and $14.3(\mathrm{c})]$ and is clearly the desired immediate successor of $Q$.

(b) Choose $Q_{0} \subset Q$ and let $\underline{Q}=\bigcup_{Q_{0} \subseteq P \subset Q} P$; then $\underline{Q} \in K_{C(X)}$ (as with $\bar{Q}$ ) and we have only to show that $\underline{Q} \neq Q$. Suppose $\underline{Q}=Q$, then for each $k=1, \ldots, n$ there is $P_{k} \in K_{C(X)}$ with $g_{k} \in P_{k}$ and $Q_{0} \subseteq P_{k} \subset Q$. Since the prime ideals containing $Q_{0}$ form a chain [GJ, 14.3(c)], we can choose $k_{0} \in\{1, \ldots, n\}$ with $P_{k} \subseteq P_{k_{0}}$ for every $k=1, \ldots, n$; hence, $g_{1}, \ldots, g_{n} \in P_{k_{0}}$. But $h \notin P_{k_{0}}$ (otherwise $h \in Q$ ) and so $P_{k_{0}}=Q$; a contradiction. We have now established the necessity of 2.4 .

If $x$ is an isolated point of $X$, then it is a $P$-point [GJ, 4L] and so $\left\{M_{x}\right\}=\tilde{\theta}_{X}{ }^{-}[x]$ is open in $K_{C(X)}$.

It follows from Stone's Representation Theorem for Boolean rings that a space $X$ is in $\mathscr{C}_{T D}$ if and only if $X=K_{A}$ for some $A \in \mathscr{R}$; that is, the contravariant functor $K: \mathscr{R} \rightarrow \mathscr{C}_{T D}$ is onto. The following corollary shows that neither $K_{C}$ nor $\zeta$ is onto $\mathscr{C}_{T D}$ and so raises the (as yet unsolved) problem of characterizing those $X \in \mathscr{C}_{T D}$ which are a $K_{C(Y)}$, or a $\zeta Y$, for some $Y \in \mathscr{T}_{3 \frac{1}{2}}$.

2.5 Corollary. Let $X$ have at least two points. Then $K_{C(X)}$, or $\zeta X$, is the one-point compactification of a discrete space if and only if $X$ is finite.

Proof. The sufficiency is obvious ( $X$ is assumed to have at least two points).

(a) Let $K_{C(X)}$ be the one-point compactification of its discrete subspace $Y$ with $P^{*} \in K_{C(X)}$ the point-at-infinity. Then each point $P \in Y$ is fixed maximal and properly contains no other prime ideals (2.4); hence, $P^{*}$ is also maximal and $X$ is a $P$-space. But $|\beta X| X \mid \leqq 1$ (recall $\tilde{\theta}_{X}$ ) and so $X$ is pseudo-compact [GJ, 6J]. The conclusion now follows as every pseudo-compact $P$-space is finite [GJ, $4 \mathrm{~K}$ ].

(b) Let $\zeta X$ be the one-point compactification of its discrete subspace $Y$ with $P^{*} \in \zeta X$ the point-at-infinity. Each point $P \in Y$ is fixed maximal and $\tilde{\theta}_{X}(P)$ is a zero-set point of $X(2.3(\mathrm{c}))$. If $P^{*}$ is also maximal, then the conclusion follows as in (a); we claim that this must be the case. Suppose that $\tilde{\theta}_{X}\left(P^{*}\right)=x \in X$ and let $X^{\prime}=X \mid\{x\} ; X^{\prime} \neq \varnothing$ as $X$ has at least two points. Then $X$ is the one-point compactification $\left(X=\tilde{\theta}_{X}[\zeta X]\right)$ of $X^{\prime}$ which is a $P$-space [GJ, 4L]. Moreover, $X^{\prime}$ is a cozero-set of $X$ (as $M_{x} \in Y$ ) and so $X^{\prime}$ is countable and discrete (as in the proof of 2.3); that is, $X=N^{*}$, the one-point compactification of $N$ with $x$ the point-atinfinity. But then the cardinality of the set of prime $z$-ideals properly contained in $M_{x}$ is $|\beta N \backslash N|[\mathrm{GJ}, 14 \mathrm{G}]$; a contradiction as this set contains only $P^{*}$. 
3. Characterizations of $\zeta X$ and its place between $\beta X_{P}$ and $\beta X$. Recall that the $H_{g, h}=h(g) \cap \ell^{c}(h)(g, h \in C(X))$ form a base for $\zeta X$; this base actually distinguishes $\zeta X$ from other compactifications of $X_{P}$.

3.1 Lemma. Let $g, h \in C(X)$. Then $h(g)=\operatorname{cl}_{\zeta X} Z(g)$ and $h^{c}(g)=\operatorname{cl}_{\zeta X} \operatorname{coz}(g)$; hence, $H_{g, h}=\operatorname{cl}_{\zeta X} Z(g) \cap \mathrm{cl}_{\zeta X} \operatorname{coz}(h)$.

Proof. It is easy to verify in general that if $A$ is a dense subset of a topological space $Y$ and $U$ is open in $Y$, then $U \subseteq \operatorname{cl}_{Y}(A \cap U)[\mathrm{K}, 1 \mathrm{G}]$. Apply this to $A=X_{P}$, $Y=\zeta X, U_{1}=h(g)$ and $U_{2}=h^{c}(g)$ to obtain one pair of inclusions; the reverse inclusions are obvious.

3.2 THEOREM (ChARACTERIZATION OF $\zeta X)$. If $Y$ is a compactification of $X_{P}$ and the collection $\left\{\operatorname{cl}_{Y} Z(g) \cap \mathrm{cl}_{Y} \operatorname{coz}(h): g, h \in C(X)\right\}$ is a base for $Y$, then there is a homeomorphism $f$ of $Y$ onto $\zeta X$ which leaves $X_{P}$ pointwise fixed.

Observe that any such $Y$ is totally-disconnected (having a base consisting of closed sets) and any such homeomorphism is unique (as $X_{P}$ is dense).

Proof. Let $Y$ be such a compactification; for $g, h \in C(X)$ we let $B_{g, h}=\mathrm{cl}_{Y} Z(g)$ $\cap \operatorname{cl}_{Y} \operatorname{coz}(h)$.

(a) If $x \in X_{P}$, then $x \in B_{g, h}$ if and only if $x \in H_{g, h}$. Hence, $B_{g_{1}, h_{1}} \cap \cdots \cap B_{g_{n}, h_{n}}=\varnothing$ if and only if $H_{g_{1}, h_{1}} \cap \cdots \cap H_{g_{n}, h_{n}}=\varnothing$.

Proof of (a). $X_{P} \cap \mathrm{cl}_{Y} Z(g)=Z(g)=X_{P} \cap \mathrm{cl}_{\zeta X} Z(g)$; likewise for $\operatorname{coz}(h)$. The first statement is now clear and the second follows as $X_{P}$ is dense in each of $Y$ and $\zeta X$.

(b) If $y \in Y$, then $H_{y}=\bigcap_{y \in B_{g, h}} H_{g, h}$ is a singleton in $\zeta X$.

Proof of (b). The collection $\left\{H_{g, h}: y \in B_{g, h}\right\}$ of closed sets in compact $\zeta X$ has the finite-intersection-property (by (a)) and so $H_{y} \neq \varnothing$. Suppose $P \neq Q \in H_{y}$; then there exist $g_{1}, h_{1} \in C(X)$ with $P \in H_{g_{1}, h_{1}}$ and $Q \notin H_{g_{1}, h_{1}}$. Then $y \notin B_{g_{1}, h_{1}}$ and so there is $B_{g_{2}, h_{2}}$ with $y \in B_{g_{2}, h_{2}}$ and $B_{g_{1}, h_{1}} \cap B_{g_{2}, h_{2}}=\varnothing$; a contradiction as $P \in H_{g_{1}, h_{1}} \cap H_{g_{2}, h_{2}}$.

We now have the function $f: Y \rightarrow \zeta X$ defined by $\{f(y)\}=H_{y}$. Precisely the same argument exhibits a function $f^{-1}: \zeta X \rightarrow Y$ with $\left\{f^{-1}(P)\right\}=\bigcap_{P \in H_{g, h}} B_{g, h}$. Observing that $y \in B_{g, h}$ implies $f(y) \in H_{g, h}$ and $P \in H_{g, h}$ implies $f^{-1}(P) \in B_{g, h}$, one sees that $f \circ f^{-1}=1_{\zeta X}, f^{-1} \circ f=1_{Y}, f^{-}\left[H_{g, h}\right]=B_{g, h}$ and $f\left[B_{g, h}\right]=H_{g, h}$.

The above argument actually shows that any $X \in \mathscr{T}_{3 \frac{1}{2}}$ which has a base $\mathscr{B}$ consisting of closed sets has at most one compactification $Y$ with the collection $\left\{\mathrm{cl}_{Y} B_{1} \cap \mathrm{cl}_{Y}\left(X \backslash B_{2}\right): B_{1}, B_{2} \in \mathscr{B}\right\}$ as a base.

Since $\beta X_{P}$ is a known compactification of $X_{P}$, the Characterization Theorem leads one to consider the collection $\mathscr{V}^{\prime}=\left\{V_{g, h}: g, h \in C(X)\right\}$, where $V_{g, h}=\mathrm{cl}_{\beta X_{P}} Z(g)$ $\cap \mathrm{cl}_{\beta X_{P}} \operatorname{coz}(h)$. A straightforward calculation (using 2.2 and [GJ, 6.9(c)]) shows that $\left(\bar{\nu}_{X}\right)^{\leftarrow}\left[H_{g, h}\right]=V_{g, h}$, where $\bar{\nu}_{X}$ denotes, as usual, the Stone-extension of $\nu_{X}: X_{P} \rightarrow \zeta X$. Thus $\mathscr{V}$ is a base for the weak topology $\tau$ on the set $\beta X_{P}$ generated by $\bar{\nu}_{X}$; also, $\left(\beta X_{P}, \tau\right)$ is coarser than $\beta X_{P}$ and so is compact. Recalling that $\bar{\nu}_{X}$ is onto $\zeta X$ (as $X_{P}$ is dense in $\zeta X$ ), it follows that $\zeta X$ is the $T_{0}$-quotient of $\left(\beta X_{P}, \tau\right)$. 
We have already noted (2.2) that $\zeta X$ is the quotient space of $\beta X_{P}$ under the equivalence relation: $M_{1} \sim M_{2} \Leftrightarrow Z\left[M_{1}\right] \cap Z(X)=Z\left[M_{2}\right] \cap Z(X)$. Consequently: $\beta X_{P}$ $\cong \zeta X$ iff $\left(\beta X_{P}, \tau\right) \cong \zeta X$ iff $Z\left[M_{1}\right] \cap Z(X)=Z\left[M_{2}\right] \cap Z(X) \Rightarrow M_{1}=M_{2}$ (where $\cong$ indicates a canonical homeomorphism, that is, one which leaves $X_{P}$ pointwise fixed). Observe that for the space $R$ of reals, $R_{P}=R_{D},\left|\beta R_{P}\right|=2^{2^{c}}$ and $|\zeta R|=2^{c}$.

4. The natural transformations $\tilde{\theta}$ and $\theta$, and the push-out $\beta$. We have already noted the continuity of $\tilde{\theta}_{X}: K_{C(X)} \rightarrow \beta X$; let $\theta_{X}$ denote the restriction of $\tilde{\theta}_{X}$ to $\zeta X$.

4.1 Proposition. $\tilde{\theta}=\left\{\tilde{\theta}_{X}\right\}_{X \in \mathscr{T}_{3 \frac{1}{2}}}$ is a natural transformation from $K_{C}$ to $\beta$; hence, $\theta=\left\{\theta_{X}\right\}_{X \in \mathscr{T}_{3 \frac{1}{2}}}$ is a natural transformation from $\zeta$ to $\beta$.

Proof. We must show that $\tilde{\theta}_{X} \circ K_{C(f)}=f \circ \tilde{\theta}_{Y}$ if $f: Y \rightarrow X$. Suppose not, then there is a $P \in K_{C(Y)}$ and $g \in C(X)$ with $g \circ f \in P$, but $\bar{f}(p) \notin \mathrm{cl}_{\beta X} Z(g)$, where $p=\tilde{\theta}_{Y}(P)$. But then $\bar{f}^{-}\left[\beta X \mid \mathrm{cl}_{\beta X} Z(g)\right]$ is a neighborhood of $p$ in $\beta Y$ which misses $Z(g \circ f)$; contrary to $p \in \mathrm{cl}_{\beta Y} Z(g \circ f)$.

A push-out in a category $A$ is a commutative diagram $m_{2} \circ m_{1}=m_{4} \circ m_{3}$ in $A$ which is best possible in the lower right corner; that is, if $m_{2}^{\prime} \circ m_{1}=m_{4}^{\prime} \circ m_{3}$, then there is a morphism $m$ unique with the property that $m \circ m_{2}=m_{2}^{\prime}$ and $m \circ m_{4}=m_{4}^{\prime}$. Push-outs are clearly unique to within equivalences; that is, if $m_{2}^{\prime} \circ m_{1}=m_{4}^{\prime} \circ m_{3}$ is also a push-out, then $m$ is an equivalence.

Let $e$ denote the natural transformation from the identity functor $I_{3 \frac{1}{2}}: \mathscr{T}_{3 \frac{1}{2}} \rightarrow \mathscr{T}_{3 \frac{1}{2}}$ to the reflector functor $\beta: \mathscr{T}_{3 \frac{1}{2}} \rightarrow \mathscr{C}$; that is, $e_{X}$ is the embedding of $X$ into $\beta X$. The natural transformations $\theta$ and $\nu$ are precisely those needed to show that $e \circ p=\theta \circ \nu$ is a push-out in Fun $\left[\mathscr{T}_{3 \frac{1}{2}}, \mathscr{T}_{3 \frac{1}{2}}\right]$, the category whose objects are functors from $\mathscr{T}_{3 \frac{1}{2}}$ to $\mathscr{T}_{3 \frac{1}{2}}$ and whose morphisms are natural transformations.

4.2 THEOREM. $e \circ p=\theta \circ \nu$ is a push-out in Fun $\left[\mathscr{T}_{3 \frac{1}{2}}, \mathscr{T}_{3 \frac{1}{2}}\right]$.

Proof. It is straightforward to show that $e_{X} \circ p_{X}=\theta_{X} \circ v_{X}$ if $X \in \mathscr{T}_{3 \frac{1}{2}}$. We let n.t. $[F, G]$ denote, as usual, the natural transformations from $F$ to $G$. Suppose $F: \mathscr{T}_{3 \frac{1}{2}} \rightarrow \mathscr{T}_{3 \frac{1}{2}}, \eta \in$ n.t. $\left[I_{3 \frac{1}{2}}, F\right]$ and $\lambda \in$ n.t. $[\zeta, F]$ are such that $\eta \circ p=\lambda \circ \nu$. We seek $\gamma \in$ n.t. $[\beta, F]$ such that $\gamma \circ \theta=\lambda, \gamma \circ e=\eta$ and whenever $\gamma^{\prime} \in$ n.t. $[\beta, F]$ so commutes, then $\gamma^{\prime}=\gamma$.

Let $X \in \mathscr{T}_{3 \frac{1}{2}}$. Since $\eta_{X} \circ p_{X}=\lambda_{X} \circ \nu_{X}$, we have $\eta_{X}[X] \subseteq \lambda_{X}[\zeta X]$; hence, $\eta_{X}^{\prime}: x \mapsto \eta_{X}(x)$ $(x \in X)$ defines a continuous function $\eta_{X}^{\prime}: X \rightarrow \lambda_{X}[\zeta X]$. Since $\lambda_{X}[\zeta X]$ is compact, we have the Stone-extension $\bar{\eta}_{X}^{\prime}: \beta X \rightarrow \lambda_{X}[\zeta X]$; hence $\gamma_{X}: p \mapsto \bar{\eta}_{X}^{\prime}(p)$ defines a continuous function $\gamma_{X}: \beta X \rightarrow F X$. We claim that $\gamma=\left\{\gamma_{X}\right\}_{X \in \mathscr{T}_{3 \frac{1}{2}}}$ is our desired natural transformation from $\beta$ to $F$.

To see that $\gamma \in$ n.t. $[\beta, F]$, let $f: Y \rightarrow X$. Since $\eta \in$ n.t. $\left[I_{3 \frac{1}{2}}, F\right]$, we have $F(f) \circ \eta_{Y}=\eta_{X} \circ f$. With this equality in mind, the two maps $\gamma_{X} \circ \bar{f}$ and $F(f) \circ \gamma_{Y}$ are seen to agree on dense $Y$ of $\beta Y$ and hence are equal.

The equality $\gamma \circ e=\eta$ is straightforward and the equality $\gamma \circ \theta=\lambda$ follows from the agreement of $\gamma_{X} \circ \theta_{X}$ with $\lambda_{X}$ on dense $\mathscr{M}_{F}(X)$. 
Finally, suppose that $\gamma^{\prime} \in$ n.t. $[\beta, F]$ with $\gamma^{\prime} \circ \theta=\lambda$ and $\gamma^{\prime} \circ e=\eta$. Then $\gamma^{\prime}=\gamma$ as $\gamma_{X}^{\prime}$ and $\gamma_{X}$ agree on dense $X$ of $\beta X$.

4.3 COROllary. $e_{X} \circ p_{X}=\theta_{X} \circ \nu_{X}$ is a push-out in $\mathscr{T}_{3 \frac{1}{2}}$.

5. The continuous and order-preserved images of $\zeta Y$. If $Y^{*}$ is a compactification of $Y$ and $f: Y^{*} \rightarrow X \in \mathscr{C}$, it is straightforward to show that $f\left[Y^{*}\right]=\operatorname{cl}_{X} f[Y]$; in particular, if $g: Y \rightarrow X$ in $\mathscr{T}_{3 \frac{1}{2}}$, then $\bar{g}[\beta Y]=\mathrm{cl}_{\beta X} g[Y]$. Moreover, if the $f$ above is also a homeomorphism, then $f\left[Y^{*} \backslash Y\right]=\mathrm{cl}_{X} f[Y] \backslash f[Y]$ [GJ, 6.11]. The following result is now clear.

5.1 Proposition. If $f: Y \rightarrow X$, then both $\zeta X$ and $\beta X$ contain a continuous and order-preserved image of $\zeta Y$; namely, $\hat{f}[\zeta Y]$ and $\theta_{X} \circ \hat{f}[\zeta Y]$, respectively. Moreover:

(a) $f[\zeta Y]=\operatorname{cl}_{\zeta X} f[Y]$, and if $f$ is also a homeomorphism, then $\hat{f}\left[\zeta Y \backslash Y_{P}\right]$ $=\operatorname{cl}_{\zeta X} f[Y] \backslash f[Y]$.

(b) $\tilde{\theta}_{X} \circ K_{C(f)}\left[K_{C(Y)}\right]=\theta_{X} \circ \hat{f}[\zeta Y]=\operatorname{cl}_{\beta X} f[Y]$, and if $f$ is also a homeomorphism and $Y$ is a $P$-space, then $\theta_{X} \circ \hat{f}\left[\zeta Y \backslash Y_{P}\right]=\operatorname{cl}_{\beta X} f[Y] \backslash f[Y]$.

An alternate proof of the first set of equalities in (b) is provided by the fact that $\tilde{\theta} \in$ n.t. $\left[K_{C}, \beta\right]$ and $\theta \in$ n.t. $[\zeta, \beta]$.

5.2 Corollary [GJ, 14F].

(a) $A$ z-ideal $P$ of $C(X)$ is prime if and only if there is an ultrafilter $\mathscr{U}$ on $X$ such that $P=\{g \in C(X): Z(g) \in \mathscr{U}\}$.

(b) The prime $z$-ideals contained in $M^{p}$ are precisely the $z$-ideals $P$ such that $P=\{g \in C(X): Z(g) \in \mathscr{U}\}$ for some ultrafilter $\mathscr{U}$ on $X$ that converges to $p$.

Proof. The mapping $\mathscr{U} \mapsto\{g \in C(X): Z(g) \in \mathscr{U}\}$ is the $\zeta$-extension of the obvious map $j: X_{D} \rightarrow X_{P}$, where $X_{D}$ is the set $X$ discretely topologized; moreover, $\hat{j}$ is onto $\zeta X(5.1(\mathrm{a}))$. Since an ultrafilter $\mathscr{U}$ on $X$ converges to $p \in \beta X$ if and only if $p=j(\mathscr{U})[\mathrm{GJ}, 6.6(\mathrm{a})]$, statement (b) holds because $\theta \in$ n.t. $[\zeta, \beta]$.

Note that statements similar to those in 5.2 can be obtained by considering the $\zeta$-extension of $p_{X}: X_{P} \rightarrow X$; in this connection, see 2.2 .

It follows directly from 5.1 that $\hat{f}$ maps $\zeta Y$ onto $\zeta X$ if and only if $f[Y]$ is dense in $X_{P}$; and that $\theta_{X} \circ \hat{f}$ maps $\zeta Y$ onto $\beta X$ if and only if $f[Y]$ is dense in $X$. Before formally stating these conclusions, however, we introduce a concept which leads, among other things, to further equivalent conditions (see 5.4 and 7.1).

Definition. Let $Y \subseteq X$. A subset $S$ of $C(X)$ is $Y$-unit-free, or $Y$-u.f., if $\left.g\right|_{Y}$ is not a unit of $C(Y)$ (equivalently, $Z(g) \cap Y \neq \varnothing$ ) for each $g \in S$. We let $\tilde{T}(Y)$ $=\left\{P \in K_{C(X)}: P\right.$ is $Y$-u.f. $\}$ and $T(Y)=\tilde{T}(Y) \cap \zeta X$.

Following is a list of properties, some obvious, which is included here for future reference; recall [GJ, 2.7] that $Z^{\leftarrow}(Z(P))=\{g \in C(X): Z(g)=Z(h)$ for some $h \in P\}$ is the smallest prime $z$-ideal containing $P \in K_{C(X)}$.

\subsection{LeMma.}

(a) Let $Y \subseteq X, S \subseteq C(X)$ and $P \in K_{C(X)}$. 
(1) If $S$ is $Y$-u.f. and $S^{\prime} \subseteq S$, then $S^{\prime}$ is $Y$-u.f.

(2) $P \in \tilde{T}(Y)$ if and only if $Z^{\leftarrow}(Z(P)) \in T(Y)$.

(3) $\tilde{T}(Y)$ is closed in $K_{C(X)}$; hence, $T(Y)$ is closed in $\zeta X$.

(b) Let $f: Y \rightarrow X$ and $S \subseteq C(X)$.

(1) $S$ is $f[Y]-u$. $f$. if and only if $g \circ f$ is not a unit of $C(Y)$ for each $g \in S$.

(2) $K_{C(f)}\left[K_{C(Y)}\right] \subseteq \tilde{T}(f[Y])$; hence, $\hat{f}[\zeta Y] \subseteq T(f[Y])$.

(3) $\mathrm{cl}_{X_{P}} f[Y]=\hat{f}[\zeta Y] \cap X_{P}=T(f[Y]) \cap X_{P}$.

Proof. (a)(3): If $P \in K_{C(X)} \mid \tilde{T}(Y)$, then there is a $g \in P$ with $Z(g) \cap Y=\varnothing$ and $\tilde{h}(g)$ is a neighborhood of $P$ which misses $\tilde{T}(Y)$. In (b)(2) note that $g \circ f \in Q \in K_{C(Y)}$ implies $g \circ f$ is not a unit of $C(Y)$. Turning to (b)(3), the first equality is clear from 5.1(a), and the second set is contained in the third by (b)(2). Finally, the third is contained in the first as every basic neighborhood $Z(g)$ of a point $x$ in $T(f[Y]) \cap X_{P}$ meets $f[Y]\left(Z(g) \cap f[Y] \neq \varnothing\right.$ as $\left.g \in M_{x} \in T(f[Y])\right)$.

Since the $G_{\delta}$-subsets of $X$ also form a base for $X_{P}$ [GJ, 1.10, 1.14(a) and 3.2(b)], it is convenient to refer to $\mathrm{cl}_{X_{P}} Y$ as the $G_{\delta}$-closure of $Y$ in $X$; similarly, $G_{\delta}$-closed and $G_{\delta}$-dense subsets of $X$ are defined (this terminology is found in [CN, 5]). It is clear that $Y$ is $G_{\delta}$-dense in $X$ if and only if $X \backslash Y$ contains no nonvoid zero-sets of $X$.

5.4 Proposition. The following assertions are equivalent for $f: Y \rightarrow X:$ (a) $\hat{f}[\zeta Y]=\zeta X$; (b) $f[Y]$ is $G_{\delta}$-dense in $X$; (c) $X \backslash f[Y]$ contains no nonvoid zero-sets of $X$; (d) $\tilde{T}(f[Y])=K_{C(X)} ;$ (e) $T(f[Y])=\zeta X$; (f) $X_{P} \subseteq \hat{f}[\zeta Y]$; (g) $X_{P} \subseteq T(f[Y])$.

Proof. (a) $\Leftrightarrow$ (b) $\Leftrightarrow$ (c): 5.1(a) and the remarks immediately above. (c) $\Leftrightarrow$ (d) $\Leftrightarrow(\mathrm{e})$ : Every nonunit is contained in some prime ideal, and 5.3(a)(2). (b) $\Leftrightarrow$ (e) and (b) $\Leftrightarrow$ (f): 5.3(b)(3).

Since $\left.h \mapsto h\right|_{X}$ is an isomorphism of $C(\beta X)$ onto $C^{*}(X)$, it is clear that $\hat{e}_{X}: \zeta X \cong \zeta(\beta X)$ if $X$ is pseudo-compact; the converse is now seen to hold by 5.4 (a) $\Leftrightarrow$ (c) and [GJ, 6I] (we will see in 6.2 that $\hat{e}_{X}$ is always a homeomorphism).

Before proceeding, however, note that if $Y \subseteq X$, then 5.1(b), 5.2(b)(2) and 5.2(a)(1) show that

$$
\operatorname{cl}_{\beta X} Y=\theta_{X} \circ \hat{\imath}[\zeta Y] \subseteq \theta_{X}[T(Y)] \subseteq\left\{p \in \beta X: O^{p} \text { is } Y \text {-u.f. }\right\},
$$

where $i: Y \rightarrow X$ is inclusion and, for $p \in \beta X, O^{p}=\left\{g \in C(X): \mathrm{cl}_{\beta X} Z(g)\right.$ is a neighborhood of $p\}$ [GJ, 7.12].

5.5 Proposition. If $Y \subseteq X$ and $i: Y \rightarrow X$ is inclusion, then

$$
\operatorname{cl}_{\beta X} Y=\theta_{X} \circ \hat{\imath}[\zeta Y]=\theta_{X}[T(Y)]=\left\{p \in \beta X: O^{p} \text { is } Y \text {-u.f. }\right\} .
$$

Proof. If $p \in \beta X$ and $O^{p}$ is $Y$-u.f., we show that $p \in \operatorname{cl}_{\beta X} Y$. Let $U$ be a neighborhood of $p$; without loss of generality, $U=Z_{\beta X}(h)$ for some $h \in C(\beta X)$ [GJ, 3.2(b)]. Then $\left.h\right|_{X} \in O^{p}$ [GJ, 7.12(a)] and so $Z\left(\left.h\right|_{X}\right)$ meets $Y$ as $O^{p}$ is $Y$-u.f.; note that $Z\left(\left.h\right|_{x}\right) \subseteq U$. 
6. $\hat{f}$ and $z$-embedding. Let $f: Y \rightarrow X$ be given. Since $\zeta Y$ is compact and $\hat{f}$ preserves order, we have: $\hat{f}$ is an (into) order-isomorphism iff $\hat{f}$ is one-to-one iff $\hat{f}$ is a (into) homeomorphism. A sufficient condition for $\hat{f}$ to be one-to-one is that $\hat{f}$ be a $z$-embedding; it is not known if this is also a necessary condition.

Definition. A subset $Y$ of $X$ is $z$-embedded in $X$ if $Z(Y) \subseteq\{Z \cap Y: Z \in Z(X)\}$; also, $f: Y \rightarrow X$ is a $z$-embedding if $f$ is a homeomorphism whose image is $z$ embedded.

Although a $C^{*}$-embedded subset is clearly $z$-embedded, the converse does not generally hold as every $z$-embedded subset of $X$ is $C^{*}$-embedded if and only if $X$ is an $F$-space $[\mathrm{H}]$. Other subsets which are always $z$-embedded are cozero-sets $\left[\mathrm{GJ}_{1}, 3.1\right]$ and Lindelöf subspaces (Jerison, $\left.\left[\mathrm{HJ}_{1}, 5.3\right]\right)$.

For $f: Y \rightarrow X$ and $P \in \zeta X$ we let $P_{f}$ denote the subset $P_{f}=\{h \in C(Y): Z(h)$ $=Z(g \circ f)$ for some $g \in P\}$ of $C(Y)$. Clearly, $P \subseteq\left\{g \in C(X): g \circ f \in P_{f}\right\}$, the latter being $\hat{f}\left(P_{f}\right)$ if $P_{f} \in \zeta Y$. But $P_{f}$ is not generally a point of $\zeta Y$; if it is, then $P \in T(f[Y])$ (as $P \subseteq \hat{f}\left(P_{f}\right)$ is $f[Y]$-u.f.) and we have seen (5.4) that $\zeta X=T(f[Y])$ if and only if $f[Y]$ is $G_{\delta}$-dense in $X$. For $P \in \zeta X$ it is easy to verify that $P$ $=\left\{g \in C(X): g \circ f \in P_{f}\right\}$ if and only if $P$ has property:

6.1 $h \in C(X), g \in P$ and $Z(h \circ f)=Z(g \circ f)$ imply $h \in P$.

As its proof indicates, the following theorem is essentially due to Mark Mandelker.

6.2 THEOREM.

(a) $f: Y \rightarrow X$ is a z-embedding if and only if the mapping of $Z(X)$ into $Z(Y)$ defined by $Z \mapsto f^{\leftarrow}[Z]$ is onto.

(b) Let $f: Y \rightarrow X$ be a z-embedding.

(1) $\hat{f}$ is an order-isomorphism and hence a homeomorphism.

(2) If $P \in \zeta X$, then $P_{f} \in \zeta Y$ if and only if $P \in T(f[Y])$. In fact, if $P \in T(f[Y])$, then $P_{f}$ is the smallest prime $z$-ideal of $C(Y)$ with $P \subseteq \hat{f}\left(P_{f}\right)$; and if $P \in \hat{f}[\zeta Y]$, then $P_{f}$ is the unique point of $\zeta Y$ with $P=\hat{f}\left(P_{f}\right)$.

(3) $\hat{f}[\zeta Y]=\{P \in T(f[Y]): P$ has property 6.1 $\}$ and $T(f[Y])=\left\{P \in \zeta X: P \subseteq \hat{f}\left(M^{p}\right)\right.$ for some $p \in \beta Y\}$.

(4) $\hat{f}[\zeta Y]=T(f[Y])$ if and only if the equality $\hat{f}\left[h\left(O^{p}\right)\right]=\left\{P \in \zeta X: P \subseteq \hat{f}\left(M^{p}\right)\right\}$ holds for each $p \in \beta Y$.

Proof. Statement (a) is $\left[\mathrm{M}_{2}\right.$, Theorem II] and shows that the proofs given in [M, I.5] generalize to obtain (b)(1) and (b)(2) with the exception of the implication $P_{f} \in \zeta Y \Rightarrow P \in T(f[Y])$ which, however, is noted above. Parts (b)(3) and (b)(4) now follow from (b)(2); specifically, for (b)(3) observe the remark incorporating 6.1 and for (b)(4) recall that $\hat{f}$ preserves order.

For future reference, we single out the result most useful to us.

6.3 Corollary. Let $f: Y \rightarrow X$ be a z-embedding such that $\hat{f}[\zeta Y]=T(f[Y])$. Then $\hat{f}$ is an order-isomorphism homeomorphism of $\zeta Y$ onto $\left\{P \in \zeta X: P \subseteq \hat{f}\left(M^{p}\right)\right.$ for 
some $p \in \beta Y\}$. In fact, $\hat{f}\left[h\left(O^{p}\right)\right]=\left\{P \in \zeta X: P \subseteq \hat{f}\left(M^{p}\right)\right\} \subseteq h\left(O^{f(p)}\right)$ if $p \in \beta Y$, with $\hat{f}\left[h\left(O_{y}\right)\right]=h\left(O_{f(y)}\right)$ if $y \in Y$.

Proof. Note that $\hat{f}\left(M^{p}\right) \subseteq M^{f(p)}$ for $p \in \beta Y$ (as $\theta \in$ n.t. $[\zeta, \beta]$ ) with $\hat{f}\left(M_{y}\right)=M_{f(y)}$ if $y \in Y(1.5)$.

Our interest is now directed toward finding $z$-embeddings with $\hat{f}[\zeta Y]=T(f[Y])$.

6.4 Proposition. If $f: Y \rightarrow X$ has $T(f[Y])$ open in $\zeta X$, then $\hat{f}[\zeta Y]=T(f[Y])$. In particular, $\hat{f}[\zeta Y]=T(f[Y])$ if either of the following two conditions holds:

(a) $X \backslash f[Y]$ contains no nonvoid zero-sets of $X$;

(b) $X \backslash f[Y]$ is a zero-set of $X$.

Proof. If $T(f[Y])$ is open, then so is $T(f[Y]) \backslash \hat{f}[\zeta Y]=T(f[Y]) \cap(\zeta X \backslash \hat{f}[Y])$; moreover, this set is contained in $\zeta X \backslash X_{P}$ (5.3(b)(3)) and so is empty as $X_{P}$ is dense. If (a) holds, then $T(f[Y])=\zeta X(5.4)$. Let (b) hold with $X \backslash f[Y]=Z(k)$; we show that $T(f[Y])=h^{c}(k)$. Since $Z(k) \cap f[Y]=\varnothing$, it is clear that $T(f[Y]) \subseteq h^{c}(k)$. Suppose $P \in h^{c}(k)$ and $P \notin T(f[Y])$; then there is $g \in P$ with $Z(g) \subseteq X \backslash f[Y]=Z(k)$, contrary to $k \notin P$.

6.5 Corollary [ $\mathrm{M}_{1}$, TheOREM IIa]. If $X$ is locally-compact and $\sigma$-compact (equivalently, $\beta X \backslash X$ is a zero-set of $\beta X$ ) and $p \in \beta X$, then the collection of prime $z$-ideals of $C(\beta X)$ contained in $\hat{e}_{X}\left(M^{p}\right)$ is order-isomorphic to the collection of prime $z$-ideals of $C(X)$ contained in $M^{p}$.

Observe that every pseudo-compact space provides a counterexample to the converse of 6.5 (see the remark immediately following 5.4).

7. The space of minimal prime ideals. The space $m(A)$ of minimal prime ideals

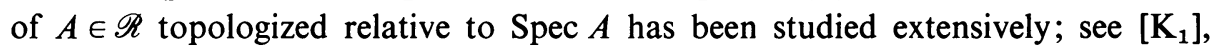
$\left[\mathrm{K}_{2}\right]$ and $[\mathrm{HJ}]$. It is clear that the topology on the set $m(A)$ relative to $K_{A}$ is finer than $m(A)$; in fact, if $A$ contains no nonzero nilpotent elements (in particular, if $A=C(X))$, then each $\tilde{h}(a) \cap m(A)$ is open in $m(A)$ [HJ, 2.3] and these relative topologies coincide. We let $m(X)$ denote the space $m(C(X))$ and note that $m(X) \subseteq \zeta X[\mathrm{GJ}, 14.7]$.

It is easy to verify [HJ, 5.3] that no proper closed subset of $m(X)$ is mapped onto $\beta X$ by $\theta_{X}$.

7.1 Proposition. The following are equivalent for $f: Y \rightarrow X:$ (a) $\theta_{X} \circ \hat{f}[\zeta Y]=\beta X$; (b) $f[Y]$ is dense in $X$; (c) $m(X) \subseteq T(f[Y])$.

Proof. (a) $\Leftrightarrow$ (b): 5.1(b). (b) $\Rightarrow$ (c): $\theta_{X}[m(X)]=\beta X$ and $\theta_{X}[T(f[Y])]=\operatorname{cl}_{\beta X} f[Y]$ (5.5). (c) $\Rightarrow$ (b): We show that the closed (5.3(a)(3)) subset $T(f[Y]) \cap m(X)$ of $m(X)$ is mapped onto $\beta X$ by $\theta_{X}$. Let $p \in \beta X=\mathrm{cl}_{\beta X} f[Y]=\theta_{X}[T(f[Y])]$; thus, there is $P \in T(f[Y])$ with $P \subseteq M^{p}$. Choose $Q \in m(X)$ with $Q \subseteq P$, then $Q \in T(f[Y]) \cap m(X)$ $(5.3(\mathrm{a})(1))$ and $\theta_{X}(Q)=p$.

Note that if $m(X) \subseteq \hat{f}[\zeta Y]$, then $f[Y]$ is dense $(5.3(\mathrm{~b})(2)$ and 7.1). 
A proof of the following theorem of Mandelker permits us to apply the preceding proposition and $\theta \in$ n.t. $[\zeta, \beta]$. Mandelker's statement, although formulated for the case $e_{Y}: Y \rightarrow \beta Y$, is equivalent to that given below (as $Y$ is $C^{*}$-embedded in $X$ if and only if $\left.i: \beta, Y=\operatorname{cl}_{\beta X} Y[\mathrm{GJ}, 6.9(\mathrm{a})]\right)$.

7.2 Theorem (MANDelker [ $\mathrm{M}_{1}$, Theorem I]). Let $Y$ be densely $C^{*}$-embedded in $X, p \in \beta Y$ and $P \in K_{C(X)}$ with $P \subseteq M^{i(p)}$, where $i: Y \rightarrow X$ is inclusion. Then $P$ is comparable to $\hat{\imath}\left(M^{p}\right)$. In fact, $P \subseteq \hat{\imath}\left(M^{p}\right)$ if and only if $P \in \tilde{T}(Y)$; while $\hat{\imath}\left(M^{p}\right) \subset P$ if and only if $P \notin \tilde{T}(Y)$.

Proof. (a): $P \subseteq \hat{\imath}\left(M^{p}\right)$ implies $P \in \tilde{T}(Y)$ (5.3(b)(2) and 5.3(a)(1)). (b): If $P \in \tilde{T}(Y)$, then $P^{\prime}=Z^{\leftarrow}(Z(P)) \in T(Y) \quad(5.3(\mathrm{a})(2))$ and $P \subseteq P^{\prime} \subseteq \hat{\imath}\left(P_{i}^{\prime}\right) \subseteq M^{i(p)}$ (6.2(b)). Hence, $i(p)=\theta_{X}\left(\hat{\imath}\left(P_{i}^{\prime}\right)\right)=i\left(\theta_{Y}\left(P_{i}^{\prime}\right)\right)$ (as $\theta \in$ n.t. $\left.[\zeta, \beta]\right)$ and $p=\theta_{Y}\left(P_{i}^{\prime}\right)$ (as $i$ is a homeomorphism, $Y$ being $C^{*}$-embedded); that is, $P_{i}^{\prime} \subseteq M^{p}$ and so $P \subseteq P^{\prime} \subseteq \hat{\imath}\left(P_{i}^{\prime}\right) \subseteq \hat{\imath}\left(M^{p}\right)$. (c): To conclude the proof we show that $P \notin \tilde{T}(Y)$ implies $\hat{\imath}\left(M^{p}\right) \subset P$. This is accomplished by showing $P$ and $\hat{\imath}\left(M^{p}\right)$ to be comparable as then (a) will yield the desired proper inclusion. Comparability is shown by choosing a $Q \in m(X)$ with $Q \subseteq P$ and verifying that $Q \subseteq \hat{\imath}\left(M^{p}\right)$ (this technique is valid by [GJ, 14.8] and is used by Mandelker in his proof). Choosing such a $Q$, we have $Q \in \tilde{T}(Y)$ (7.1) and $Q \subseteq M^{i(p)}$; consequently, $Q \subseteq \hat{\imath}\left(M^{p}\right)$ (by (b), replacing $P$ with $Q$ ).

Note that if $Y$ is $z$-embedded but not $C^{*}$-embedded, then we can choose $p \neq q$ in $\beta Y$ with $i(p)=i(q)$; hence, $\hat{\imath}\left(M^{q}\right) \subseteq M^{i(q)}=M^{i(p)}$ (the first inclusion as $\theta \in$ n.t. $[\zeta, \beta]$ ) but $\hat{\imath}\left(M^{q}\right)$ is not comparable to $\hat{\imath}\left(M^{p}\right)$ (as $\hat{\imath}$ is an order-isomorphism).

7.3 Corollary. Let $Y$ be densely z-embedded in $X$. Then $Y$ is $C^{*}$-embedded if and only if $p \in \beta Y$ and $P \subseteq M^{i(p)}$ in $K_{C(X)}$ imply that $P$ is comparable to $\hat{\imath}\left(M^{p}\right)$.

7.4 Proposition. Let $f: Y \rightarrow X$.

(a) $\theta_{X} \circ \hat{f}[m(Y)]=\mathrm{cl}_{\beta X} f[Y]$.

(b) If $f[Y]$ is dense and $\hat{f}[m(Y)] \subseteq m(X)$, then $\hat{f}[m(Y)]=m(X)$.

Proof. (a): 5.1(b) provides the inclusion $\theta_{X} \circ \hat{f}[m(Y)] \subseteq \mathrm{cl}_{\beta X} f[Y]$ (as $m(Y) \subseteq \zeta Y$ ) as well as the reverse inclusion (for $p=\theta_{X} \circ \hat{f}(P) \in \mathrm{cl}_{\beta X} f[Y]$ choose $Q \in m(Y)$ with $Q \subseteq P$ ). (b): The argument of (a) now shows that $\theta_{X}[\hat{f}[\zeta Y] \cap m(X)]=\operatorname{cl}_{\beta X} f[Y]$ (as $\hat{f}[m(Y)] \subseteq m(X)$; i.e., $\hat{f}(Q) \in m(X)$ for the $Q$ chosen in (a)) which is now all of $\beta X$; hence, $m(X) \subseteq \hat{f}[\zeta Y]$ (as no proper closed subset of $m(X)$ maps onto $\beta X$ ).

To prove the following lemma we use: $P \in K_{C(X)}$ is in $m(X)$ if and only if $g \in P$ implies the existence of $h \notin P$ with $g \cdot h=0$ [HJ, 1.1].

7.5 Lemma. Let $f: Y \rightarrow X$ be a z-embedding. Then $\hat{f}[m(Y)] \subseteq m(X)$ if either one of the following two conditions holds:

(a) $f[Y]$ is dense;

(b) $f[Y]$ is a cozero-set of $X$.

Proof. Let $Q \in m(Y)$ and $g \in \hat{f}(Q)$; we seek $g^{\prime} \notin \hat{f}(Q)$ with $g \cdot g^{\prime}=0$. Choose $h \notin Q$ with $h \cdot(g \circ f)=0$ (as $g \circ f \in Q \in m(Y)$ ) and choose $t \in C(X)$ with $Z(h)$ 
$=Z(t \circ f)$ (by 6.2(a)). Note that $t \notin \hat{f}(Q)$ (as $h \notin Q)$ and $f[Y] \subseteq Z(t \cdot g)$ (as $h \cdot(g \circ f)$ $=0)$. Thus, if $f[Y]$ is dense we can let $g^{\prime}=t$; and if $X \backslash f[Y]=Z(k)$ we can let $g^{\prime}=k \cdot t$.

7.6 THEOREM. If $f: Y \rightarrow X$ is a dense z-embedding, then $\hat{f}$ restricted to $m(Y)$ is a homeomorphism of $m(Y)$ onto $m(X)$.

Proof. 6.2(b)(1), 7.4(b) and 7.5.

Observe that denseness cannot be dropped from the hypothesis of this theorem (7.1 (b) $\Leftrightarrow$ (c) and 5.3(b)(2)). Also note that $f: Y \rightarrow X$ can be onto without $\hat{f}: m(Y) \cong m(X)$; for example, let $X \neq X_{P}$ and $f=p_{X}: X_{P} \rightarrow X$.

7.7 Corollary.

(a) [HJ, 5.2] $\hat{e}_{X}$ restricted to $m(X)$ is a homeomorphism of $m(X)$ onto $m(\beta X)$.

(b) $\hat{\imath}$ restricted to $m(Q)$ is a homeomorphism of $m(Q)$ onto $m(R)$, where $i$ denotes the inclusion of the rational numbers $Q$ into $R$.

(c) If $X$ is countable, then $\hat{v}_{X}: \beta X_{D} \cong m(\zeta X)$.

Proof. Both $i$ and $\nu_{X}$ are $z$-embeddings as countable spaces are Lindelöf. In (c), note that a countable $P$-space is discrete [GJ, $4 \mathrm{~K}$ ].

8. Some final applications. Suppose a topological space $X$ has a subset $A$ which is closed, contains only isolated points of $X$, and is disjoint from a subset $A^{\prime} \subseteq X$ whose union with $A$ is dense in $X$. Then, it is straightforward to verify that $\mathrm{cl}_{\mathrm{X}} A^{\prime}=X \backslash A$.

8.1 TheOrem. Let $f: Y \rightarrow X$ be such that $A=X \backslash f[Y]$ is finite and contains only zero-set points of $X$.

(a) $\hat{f}[\zeta Y]=\zeta X \mid\left\{M_{a}: a \in A\right\}$.

(b) If $f$ is a homeomorphism, then $f$ is a z-embedding with $\hat{f}[\zeta Y]=T(f[Y])$ (so that the hypothesis of 6.3 is satisfied); also $\hat{f}\left[\zeta Y \backslash Y_{P}\right]=\zeta X \backslash X_{P}$.

(c) If $f$ is a homeomorphism and $\mathrm{cl}_{\beta X} f[Y] \subseteq X$, then the collection of free maximal ideals of $C(Y)$ is mapped one-to-one onto the collection of prime z-ideals maximal among those properly contained in the $M_{a}(a \in A)$.

(d) If $f$ is a homeomorphism and no point of $A$ is isolated in $X$, then $\hat{f}$ restricted to $m(Y)$ is a homeomorphism onto $m(X)$.

Proof. Letting $A^{\prime}=f[Y], A$ and $A^{\prime}$, viewed as subsets of $\zeta X$, are as in the preceding remark (2.3(c) and $X_{P}$ is dense); hence, (a) holds by 5.1(a). Observe that $X \backslash f[Y]$ is a zero-set of $X$; hence, (b) holds by 6.4(b) and 5.1(a), respectively. In (c), since $\bar{f}(p) \in \operatorname{cl}_{\beta X} f[Y] \backslash f[Y]$ (by the opening remarks of $\S 5$ ), the conclusion follows from (a) and 6.3. Finally, in (d), $f[Y]$ is now dense in $X$ and 7.6 applies.

Observe that if the hypotheses of both (c) and (d) are satisfied, then $X$ must be compact.

We now have a rather complete description of $\zeta([0,1])$ in terms of $\zeta R$ by taking a homeomorphism $f: R \rightarrow[0,1]$ with $f[R]=(0,1)$ and observing that all the various 
hypotheses of 8.1 are satisfied. Although the two isolated points $M_{0}$ and $M_{1}$ can be topologically absorbed by $\zeta([0,1])$ (which contains uncountably many such points) to obtain $\zeta R \cong \zeta([0,1])$, one loses the order-isomorphism properties of $\hat{f}$ in doing so.

Likewise, the inclusion $i$ of $N$ into $N^{*}=N \cup\{\omega\}$, its one-point compactification, yields the known [GJ, 14G] prime $z$-ideal structure of $\zeta N^{*}$ in terms of $\beta N$; here, the minimalness of the prime $z$-ideals properly contained in $M_{\omega}$ follows from the noncomparability of the (maximal) prime ideals in $\zeta N \backslash N=\beta N \backslash N$ (or from 7.6).

Finally, insight is gained whenever one has a one-to-one sequence $s: N \rightarrow X$ such that $S=s[N]$ contains no cluster points of $S$ in $X$ (equivalently, $s$ is a homeomorphism), for then: (1) $\theta_{X} \circ \hat{S}[\beta N]=\operatorname{cl}_{\beta X} S$ and $\theta_{X} \circ \hat{s}[\beta N \backslash N]=\operatorname{cl}_{\beta X} S \backslash S$ (5.1(b)); and (2) $\hat{s}$ is an order-isomorphism homeomorphism ( $S$ is Lindelöf and 6.2).

\section{REFERENCES}

[B] N. Bourbaki, Éléments de mathématiques. Fasc. 27: Algèbre commutative. Chapitre 2: Localisation, Actualités Sci. Indust., no. 1290, Hermann, Paris, 1961. MR 36 \#146.

[CN] W. W. Comfort and S. Negrepontis, Extending continuous functions on $X \times Y$ to subsets of $\beta X \times \beta Y$, Fund. Math. 59 (1966), 1-12. MR 34 \#782.

[GJ] L. Gillman and M. Jerison, Rings of continuous functions, Van Nostrand, Princeton, N. J., 1960. MR 22 \#6994.

[GJ $\left.\mathbf{J}_{1}\right]-$, Quotient fields of residue class rings of function rings, Illinois J. Math. 4 (1960), 425-436. MR 23 \#A2038.

[H] A. W. Hager, $C$-, $C^{*}$-, and z-embedding, (to appear).

[HJ] M. Henriksen and M. Jerison, The space of minimal prime ideals of a commutative ring, Trans. Amer. Math. Soc. 115 (1965), 110-130. MR 33 \#3086.

[HJ $\left.\mathrm{H}_{1}\right]$ M. Henriksen and D. G. Johnson, On the structure of a class of archimedean latticeordered algebras, Fund. Math. 50 (1961/62), 73-94. MR 24 \#A3524.

[K] J. L. Kelley, General topology, Van Nostrand, Princeton, N. J., 1955. MR 16, 1136.

[K $\left.\mathbf{K}_{1}\right]$ J. Kist, Minimal prime ideals in commutative semigroups, Proc. London Math. Soc. (3) 13 (1963), 31-50. MR 26 \#1387.

$\left[\mathbf{K}_{2}\right]-$ Compact spaces of minimal prime ideals, Math. Z. 111 (1969), 151-158.

[M] M. Mandelker, Prime z-ideal structure of $C(R)$, Fund. Math. 63 (1968), 145-166.

$\left[\mathbf{M}_{1}\right]-$, Prime ideal structure of rings of bounded continuous functions, Proc. Amer. Math. Soc. 19 (1968), 1432-1438. MR 37 \#6761.

$\left[\mathbf{M}_{2}\right] \longrightarrow, F^{\prime}$-spaces and z-embedded subspaces, Pacific J. Math. 28 (1969), 615-621.

$\left[\mathbf{M}_{3}\right]$ R. Montgomery, Structures determined by the prime ideals of rings of continuous functions, Dissertation, Clark University, Worcester, Mass., 1969.

Clark University, WORCESTER, MASSACHUSETTS 